\title{
The 10th Japan Bioanalysis Forum Symposium conference: a report
}

Yoshihisa Sano*,1, Yoshiaki Ohtsu², Harue Igarashi ${ }^{3}$, Jun Hosogi ${ }^{4}$, Junji Komaba ${ }^{5}$, Masanari Mabuchi $^{6}$, Takeru Yamaguchi ${ }^{7}$, Hiroshi Kamimori ${ }^{8}$, Tomoko Arakawa9 ${ }^{9}$, Takahiro

Nakamura $^{10}$, Keiko Nakai ${ }^{11}$, Hisanori Hara ${ }^{12}$, Masaaki Kakehi ${ }^{13}$, Sakiko Suzuki ${ }^{1}$, Hitoshi

Uchiyama $^{14}$, Yosuke Kawai ${ }^{15}$, Akemi Nagao ${ }^{16}$ \& Yumi Nishiguchi ${ }^{17}$

${ }^{1}$ Sunplanet Co., Ltd, Tokodai, Tsukuba, Ibaraki, Japan

${ }^{2}$ Astellas Pharma, Inc., Tsukuba, Ibaraki, Japan

${ }^{3}$ GlaxoSmithKline KK, Tokyo, Japan

${ }^{4}$ Kyowa Kirin Co., Ltd, Nagaizumi, Shizuoka, Japan

${ }^{5}$ Ono Pharmaceutical Co., Ltd, Tsukuba, Ibaraki, Japan

${ }^{6}$ Mitsubishi Tanabe Pharma Corporation, Yokohama, Japan

${ }^{7}$ Sumika Chemical Analysis Service, Ltd, Osaka, Japan

${ }^{8}$ Shionogi \& Co., Ltd, Osaka, Japan

${ }^{9}$ Pfizer R\&D Japan GK, Tokyo, Japan

${ }^{10}$ Shin Nippon Biomedical Laboratories, Ltd, Kainan, Wakayama, Japan

${ }^{11}$ LSI Medience Corporation, Kamisu, Ibaraki, Japan

${ }^{12}$ Novartis Pharma AG, Basel, Switzerland

${ }^{13}$ Takeda Pharmaceutical Company Limited, Fujisawa, Kanagawa, Japan

${ }^{14}$ Towa Pharmaceutical Co., Ltd, Kyoto, Japan

${ }^{15}$ Otsuka Pharmaceutical Co., Ltd, Otedori, Chuo-ku, Osaka, Japan

${ }^{16}$ Japan Tobacco, Inc., Osaka, Japan

${ }^{17}$ CMIC Pharma Science Co., Ltd, Hyogo, Japan

*Author for correspondence: Tel.: +81 29847 5637; Fax: +81 29847 5613; y2-sano@hhc.eisai.co.jp

All authors are 10th Anniversary JBF Symposium project members

The Japan Bioanalysis Forum Symposium was held on 12-14 February 2019 (Yokohama, Japan), in celebration of its 10th anniversary, and over 370 participants from pharmaceutical industries, contractors, academia and regulatory authorities from home and abroad came together in Yokohama. The 3-day symposium particularly aimed to foster collaboration with the scientists surrounding bioanalysts, according to the theme 'Open to the Public.' The symposium also included a broad range of pioneering programs, such as lectures by speakers from DMPK/metabolomics fields, discussions of future bioanalysis and poster presentations by publicly offered presenters as well as the regular ones we had organized. This report summarizes the major topics as a conference report.

First draft submitted: 20 June 2019; Accepted for publication: 8 July 2019; Published online:

8 August 2019

Keywords: bioanalysis $\bullet$ biomarker $\bullet$ clinical pharm $\bullet$ data integrity $\bullet$ discussion group $\bullet$ drug-drug interaction $\bullet$ $\mathrm{ICH} \mathrm{M10} \mathrm{•} \mathrm{JBF} \mathrm{•} \mathrm{LBA} \mathrm{•} \mathrm{LC-MS/MS} \mathrm{•} \mathrm{microsampling}$

Since the first Japan Bioanalysis Forum (JBF) Symposium in 2011, which was the first-ever scientific meeting focused on regulated bioanalysis in Japan, the JBF continuously offers a room, for discussing bioanalytical matters/questions from Japanese scientists. The symposium celebrated its 10th anniversary recently, in February 2019.

During these years, the scope of drug discovery and development has drastically changed. The technologies being employed in the bioanalysis of new modalities, such as the so-called large and middle molecules, have been concomitantly developed. Accordingly, bioanalysts need to expand their views outward in collecting relevant information and are required to upgrade their skills and knowledge.

Thus, we held the 10th symposium with the theme 'Open to the Public' aiming to foster practical collaboration with the scientific fields surrounding bioanalysis, including DMPK, Tox and Clinical Pharm. In addition, special events to celebrate the 10th anniversary were organized. 
This report provides a summary of the highlights of the 10th JBF Symposium (Yokohama, Japan, 12-14 February 2019). Presentation materials of the symposium are available on the JBF website [1].

\section{Keynote lecture/special lecture (days 1 \& 2)}

Tetsuya Terasaki (Tohoku University, Sendai, Japan) delivered a keynote lecture entitled, 'New Horizon of Drug Discovery and Development: Quantitative Proteotyping Accelerated by Reliable Peptide Search (rPS) Engine'. Takeshi Bamba (Kyushu University, Fukuoka, Japan) gave a special lecture entitled, 'Recent trends and future challenges of metabolome analysis'.

\section{Future of bioanalysis (days 1 \& 2)}

Shinobu Kudoh (Yokogawa Electric, Tokyo, Japan) provided a roundup of bioanalysis from the middle of the 1980s to the establishment of JBF. This event was an ideal opportunity for all attendees, especially young bioanalysts, to understand the accomplishments, technological advancement and remaining issues in bioanalysis. Subsequently, industry experts held a panel discussion on innovation in technologies and services. A number of ideas on the use of artificial intelligence, chat bots, virtual reality and augmented reality in bioanalysis were proposed. In addition, many expressed their desire for advancements in mass spectrometry and ligand binding assays (LBAs). Afterward, Philip Timmerman (European Bioanalysis Forum [EBF]) presented five challenges for the 21st-century bioanalysts. In the presentation, Timmerman focused on the following areas: biomarkers, outsourcing, regulations, accepting exceptions and technology. Last, Yoshiaki Ohtsu, as the JBF deputy representative, discussed the impact of corporate trends, demographic dynamics, changes in social norms and regulations and transition to Society 5.0 [2] on bioanalysis in Japan, and encouraged the attendees to adopt a broad vision.

\section{Update on the validation of analytical methods for biomarkers (day 1)}

Yoshiro Saito (National Institute of Health Sciences, Kanagawa, Japan) introduced the Japan Agency for Medical Research and Development research group, which started drafting the points to consider document on biomarker assay validation in Japan, and then explained their basic principles, current discussion and future perspective. Akira Wakamatsu (GlaxoSmithKline, Tokyo, Japan) proposed a strategy for selecting surrogate matrix in the quantitation of endogenous substances discussed in a JBF discussion group (DG; DG2015-15). As candidates, a buffer containing protein (e.g., bovine serum albumin, casein) for LBAs and a simple solvent (e.g., water, buffer) for LC-MS were preferred [3]. Takashi Miyayama (Chugai, Kanagawa, Japan) presented three cases of biomarker assay platforms, including method validation, in clinical studies. Miyayama mentioned that the acceptance criteria of Bioanalytical Method Validation Guidelines should be considered if biomarkers closely relate to the drug target and the results are used as quantitative values. Akihide Tsujimoto (Mitsubishi Tanabe, Yokohama, Japan) explained a biomarker method validation using immunoassay platform (single and multiplex) in the exploratory and/or decision-making stage, demonstrating a new index of 'total variability', which means the total of biological variability and analytical error, and stated that biomarker responses should be larger than the total variability. In the panel discussion, Hisao Shimizu (Takeda, Kanagawa, Japan) and Toshihiro Oguma (Daiichi Sankyo, Tokyo, Japan) joined to the presenters, and they reached a consensus in the following:

- If authentic standards of proteins were not available, then recombinants were sufficient in an early clinical stage and useful even in later stages;

- Even if the expected A\&P might not meet set criteria, the biomarker analysis should be continued considering the balance of detection variability and required response;

- Differentiate the criteria between exploratory and proof-of-concept studies;

- Need to discuss deeply with stakeholders and overlap each function.

\section{Follow-up session for biomarker assays (day 3)}

What should we do for 'better' biomarker evaluation?

Bioanalysts make efforts in effectively evaluating biomarkers in drug development under tight and floating deadlines. This session aimed to approach the best practice for the above and promote the sharing of experiences/suggestions among analysts. Yoshiaki Ohtsu (Astellas, Ibaraki, Japan), Toshihiro Oguma, Hisao Shimizu, Akihide Tsujimoto, Takahiro Nakamura (Shin Nippon Biomedical Laboratories), Takashi Miyayama, Yutaka Yasuda (Toray, Kanagawa, 
Japan), Akira Wakamatsu, Harue Igarashi (GlaxoSmithKline) and Makoto Niwa (Nippon Kayaku, Tokyo, Japan) were seated at the table as the panelists. The following four cases were discussed:

- Termination of critical reagents' supply;

- Sourcing rare matrices;

- Budget management in outsourcing preliminary studies;

- Analytical methods transfer.

The consensus they reached in the first two cases were as follows: some backup plans for the assay method is required for long-term drug development; it is preferable to restart analyzing all samples by a new method rather than switching the method halfway and additionally conducting a cross-validation, from the viewpoint of data consistency; in early clinical trials, the backup method should be considered for replacement in further phases (for the first case); the supply of blank cerebrospinal fluid of sufficient quality (i.e., free of blood contamination) increases with the market needs; the quality and collection method of matrices are paramount; consulting with experienced CROs may be beneficial (for the second case).

\section{Hot topics in the bioanalysis of antibody drugs (days 2 \& 3 )}

Boris Gorovits (Pfizer, MA, USA) overviewed the points to consider in the development of antibody-drug conjugation (ADC). Based on the heterogeneity of ADC molecule species with various drug-to-antibody ratios, the determination of multiple analytes, such as free drugs and conjugated antibodies, help elucidate the complexity of PK and disposition of ADC. Moreover, LBA, LC-MS, and the combination of both methods should be properly used depending on the information sought. Ichio Onami (Chugai) presented recent approaches for developing bioanalytical methods to determine total and/or free therapeutic antibodies. When developing an assay for free antibodies, multiple factors that can potentially affect the equilibrium between free and total antibodies should be assessed, such as incubation time, sample dilution, and concentration of capture reagents. The presentation demonstrated that the free analyte QC concept is helpful for assay qualification [4]. Kazuhiro Miya (Chugai) introduced their experiences and current perspective on ADA assay strategy over the last decade (total of 19 programs: seven approved, 12 in development). They have mainly used electro-chemiluminecence or ELISA for screening/confirmatory assays. Neutralizing assays are ready for approximately one third of the projects. The significance of acid dissociation to improving drug tolerance is increasing. He also emphasized that the regulatory requirements change yearly and new discussions emerge in this field.

\section{Utilizing endogenous biomarkers for drug-drug interaction evaluation \& bioanalytical method validation (day 2)}

This session was held as a joint session of JBF and The Japanese Society for the Study of Xenobiotics. Hiroyuki Kusuhara (the University of Tokyo, Tokyo, Japan) presented that plasma concentrations of coproporphyrin I, direct bilirubin and glycochenodeoxycholic acid-3-glucuronide are elevated by the administration of rifampicin, an inhibitor of hepatic transporter OATP1B1. This result indicated that these endogenous substrates could be drugdrug interaction (DDI) biomarkers for OATP1B1. In utilizing the PBPK models incorporating these biomarkers, DDI risk can be predicted in the early clinical stage. Jianing Zeng (Bristol-Myers Squibb, NJ, USA) discussed method validation strategies for the bioanalysis of biomarkers for transporters, including coproporphyrin I. Fit-forpurpose bioanalytical strategies were implemented to support all stages of DDI evaluation. She emphasized that both bioanalytical and practical aspects need to be taken into consideration to utilize the biomarkers.

$4 \beta$-hydroxycholesterol ( $4 \beta$-OHC) has been reported to be a useful biomarker for CYP3A activity. Yosuke Suzuki (Meiji Pharmaceutical University, Tokyo, Japan) mentioned that the plasma concentration of $4 \beta-\mathrm{OHC}$ is significantly elevated in patients with end-stage renal disease after kidney transplantation, which suggests that CYP3A activity is restored by renal transplantation. Yuri Takeda (Shionogi, Osaka, Japan) presented that the $4 \beta-\mathrm{OHC}$ to cholesterol ratio could be a useful biomarker to predict DDI risk mediated by CYP3A. She explained that chromatographic resolution from isobaric interferences and selection of the surrogate matrices are key points in the development of LC-MS/MS methods for the biomarkers.

\section{Update of ICH-M10 \& US FDA guidance (day 2)}

Steve White (EBF) presented EBF's previous discussion and future plans related to ICH-M10. The activities related to the US FDA 2018 Bioanalytical Method Validation Guideline were also introduced [5]. Six topics (Use of Fresh 
QCs, Fit for Purpose, Documenting Method Development, Cross Validation, ISR and Fixed Combinations and Specific Drug Regimens) were selected based on their experiences in dealing with the new guidance over the last 6 months. The activity resulted in consensus on certain topics, although different opinions remained on others.

\section{Technologies supporting bioanalysis (day 3)}

Bioanalysis of macromolecular drugs by LC-MS

Although bioanalysis of therapeutic proteins is generally conducted using LBA, LC-MS has also been employed recently, and its application is increasing gradually. Surinder Kaur (Genentech, CA, USA) introduced the development of new hybrid bioanalytical strategies involving affinity capture (IA) and LC-MS/MS as alternative approaches for protein bioanalysis. Signature peptides generated by digesting proteins can be used as surrogates for quantifying therapeutic proteins. Kaur had quantified many therapeutic proteins by IA-LC-MS/MS and LBA, and the results obtained using both methods were comparable. Kaur also emphasized that complementary and multiple method platforms using LC-MS/MS and LBA can provide advantages in nonclinical and clinical bioanalysis as molecular complexity increases.

Yuchen Sun (National Institute of Health Sciences, Kanagawa, Japan) introduced their studies regarding bioanalytical methods of antisense oligonucleotide therapeutics with LC-MS. Sun's team developed a quantification method of mipomersen in rat plasma by extracting mipomersen from rat plasma, separating the same using ion pair chromatography and detecting by high-resolution mass spectrometry. They optimized the method and obtained good results. Sun stated that this method can also be useful for the bioanalysis of other antisense oligonucleotide therapeutics.

\section{Advanced technology of bioanalysis in the drug discovery stage}

Junya Kato (Ono Pharmaceutical, Osaka, Japan) presented two application examples using a high-resolution mass spectrometer for early-phase ADME evaluation. One was in vitro metabolic stability assay that could concomitantly identify metabolites as a high-throughput screening. The other was a pharmacokinetic assessment of test compounds mixed in a cocktail replacing the conventional method employing triple-quad mass spectrometers.

Yosuke Kotani (Shin Nippon Biomedical Laboratories) introduced enzyme-linked oligosorbent assay to quantitate nucleic acid molecules by electro-chemiluminecence or Gyrolab. The superiority of two oligonucleotide probes, namely, a template probe for immobilization and a ligation probe for detection, was indicated.

Yukari Tanaka (Shionogi, Osaka, Japan) presented an imaging MS (IMS) technique for drug distribution analysis in brain tissues. They developed a reproducible and quantitative matrix-assisted laser desorption/ionization IMS (MALDI-IMS) method for Epertinib (S-222611), which is a potent, reversible and selective tyrosine kinase inhibitor of the EGFR, HER2 and human EGFR4. The results indicated that the MALDI-IMS method could be useful in selecting prospective drug candidates at the early stages of drug discovery and development.

\section{Synergy with scientific areas surrounding bioanalysis (day 3)}

\section{Collabo session with clinical pharmacology: collaboration in early clinical trials}

Yuji Kumagai (Kitasato University, Tokyo, Japan) presented the advantage of bioanalysis in early clinical trials. Securing the safety of participants is the primary interest in conducting early clinical trials. The tragedy that occurred during the first-in-human trial in France cannot be forgotten; it might have been avoided if drug concentrations were monitored carefully. The Japanese Society of Clinical Pharmacology and Therapeutics (Tokyo, Japan) published a checklist for early clinical trials [6] in 2018, in which pharmacokinetic information is essential for step-up criteria in first-in-human trials. Biomarkers are also important for detecting adverse drug reactions before irreversible damage occurs; the quest for new safety markers is also highly expected. In the panel discussion, Shin Irie and Hinako Uchimaru (Souseikai, Fukuoka, Japan), Masaaki Kakehi (Takeda) and Yosuke Kawai (Otsuka) served as panelists. Discussion was held on the quick turnaround of drug concentration data, issue of safety biomarkers and collaboration between clinical pharmacologists and bioanalysts. To deliver the data in the required timing and format, communication is critical. To establish safety biomarkers, the accumulation of evidence and cross-company collaboration would be necessary. The mutual understanding of needs and expertise would be essential and could lead to innovative approaches in clinical development. 
Collabo session with Japan Society of Quality Assurance: perspective of data integrity for electronic raw data on LC-MS/MS

Study Group 3 of the Japan Society of Quality Assurance is the Good Laboratory Practice Division. They investigate and propose detailed procedures that ensure the reliability of electronic data and computerized systems. Yoshihisa Kusakawa (CMIC Pharma Science, Hyogo, Japan) presented a recommendation for the equipment and systems classification and procedures for operation and management in each category, and demonstrated an operation management method to ensure data integrity using LC-MS/MS systems, in which raw data could be recorded electronically. Misae Ito (Chugai) introduced the use of appropriately qualified/validated equipment to improve data reliability and highlighted detailed viewpoints of the Quality Assurance Unit on the inspection of sample measurement using LC-MS/MS.

\section{Collabo session with toxicity evaluation: practicing microsampling}

The ICH S3A Q\&A focusing on microsampling was finalized in November 2017. The notification is ready for issuance in Japan after other countries. The session was set up in preparation for increases in its practical use. In advance of publication in Japan, Jihei Nishimura (Pharmaceuticals and Medical Devices Agency, Tokyo, Japan) explained the Japanese version of ICH S3 Q\&A. Yoshiro Saito updated the outline of microsampling mainly used in foreign countries. From the perspective of a toxicologist, Hirohiko Ohtsuka (Axcelead Drug Discovery Partners, Inc., Kanagawa, Japan) and Yui Akagawa (LSI Medience, Ibaraki, Japan) presented examples of the actual toxicity evaluation and TK results, focusing on sampling time, site, and devices. A panel discussion was then held by the presenters and additional toxicologists as panelists, focusing on the reproducibility of toxicity evaluation (e.g., the effect of blood collection on the physiological condition of animals) and possible influences on pharmacokinetics.

\section{JBF discussion groups (days 2 \& 3)}

The DG promotion committee (seven members from industries, led by Yoshihisa Sano of Sunplanet, Tsukuba, Japan) organized this session. The five DGs (DG35 to 39) presented their achievements after a year of discussion on the focused topics and exchanged their views with attendees on a broad range of bioanalytical issues in the poster session in the evening of day 2 and the morning of day 3 .

DG35 held discussions on the topic: 'Revisiting accuracy and precision (A\&P) criteria' with emphasis on the early phase metabolite testing workflow. The group consisted of nine members (leader: Makoto Niwa, Nippon Kayaku). They focused on the applicability of the surrogate standard calibration curve (i.e., analogous standard for newly detected metabolites) with response factor correction. The idea of uncertainty in evaluating the impact of this procedure quantitatively was introduced. The extent of uncertainty with typical surrogate standard approaches was shared with the attendees.

DG36 discussed the topic of 'Quantitative analysis of oligonucleotide therapeutics by LC-MS'. The group consisted of nine members (leader: Yoshiharu Hayashi, CMIC Pharma Science, Hyogo, Japan). They focused on the five themes in which the DG members were interested: sample preparation, instruments, internal standard, validation, and comparison with LBA. They likewise presented an overview of their discussion. Moreover, they surveyed the current situation of the quantification of oligonucleotide therapeutics using LC-MS and then shared the results.

DG37 discussed the topic of 'Automated sample preparation in LC-MS bioanalysis'. The group consisted of ten members (leader: Takumi Noda, Ono Pharmaceutical, Osaka, Japan). They thought their previous presentation, DG32 at the 9th JBF Symposium (6-8 February 2018, Tokyo, Japan), was limited to sharing the usage of automation in Japan and could not indicate workable countermeasures on site. This time, the DG considered these aspects for the specific problems in regulated bioanalysis at the time of the introduction of automation. They also presented examples of automation in not only regulated bioanalysis but also early drug discovery studies.

DG38 discussed the topic of 'Parallelism in ligand binding assay (LBA)'. The group consisted of five members (leader: Hiroyuki Shimizu, Mitsubishi Tanabe, Yokohama, Japan). They noted that the objectives and methods of evaluating parallelism for biomarker analysis are different from those of PK (TK), and that setting an appropriate acceptance criterion based on each analysis purpose is important. The anticipated issues, and the approaches to solve the issues, were also discussed. Recommended processes of evaluating parallelism in LBA were shared with the attendees for both biomarker analysis and PK (TK) based on their discussion.

DG39 discussed the topic of 'Failure and trouble cases in LBA and their solutions'. The group consisted of six members (leader: Tetsu Saito, Astellas Pharma, Tsukuba, Japan). They listed various failures and troubles in 
the respective steps of LBA procedures often faced by analysts, and then discussed solutions and recommended methods. They also surveyed LBA analysts to collect diverse opinions and actual cases. The collected cases of troubles and their countermeasures were shared with the attendees to help them solve problems regarding LBA in bioanalysis.

\section{Other events to celebrate the 10th anniversary}

A big chronology panel $\left(200 \times 80 \mathrm{in}^{2}\right)$ was prepared and displayed in the hall of the poster sessions and the 8 -year history of JBF was commemorated by participants. Poster presentations by publicly offered presenters were planned, and approximately 30 speakers applied to this first-ever event. Among them, three excellent presentations were awarded by JBF. Participants also enjoyed the so-called stamp-rally: they went around the booths of the symposium sponsors and received fabulous gifts from the sponsors. Meanwhile, two basic lectures on quantitative LC-MS/MS and LBA, convened prior to the formal symposium programs, were particularly welcomed by young scientists and new practitioners.

\section{Conclusion}

In this decade, bioanalysis has expanded its roles in the field of drug discovery and development along with the ever-widening range of assay technologies. Its relation with the surrounding areas, such as DMPK, Tox and Clinical Pharm, has grown more critical to accomplishing its goals. Collaboration with these scientific fields would be crucially required anywhere bioanalysts play an active part.

We have successfully completed the 10th anniversary JBF symposium, through which we offered a room, for discussing bioanalytical matters/questions, according to the theme 'Open to the Public'. The symposium hosted remarkable sessions, presenters and special events. We anticipate that we could continuously invite all stakeholders, from bioanalysts to scientists in areas related to bioanalysis, and spend valuable time for interactive discussions toward the 'beginning of the new era', which is the main concept of the next (11th) symposium.

The 11th JBF symposium will be held in Tokyo, Japan, on 25-27 February 2020. We hope to meet with all who share an interest in the future stage of bioanalysis.

\section{Financial \& competing interests disclosure}

The authors have no relevant affiliations or financial involvement with any organization or entity with a financial interest in or financial conflict with the subject matter or materials discussed in the manuscript. This includes employment, consultancies, honoraria, stock ownership or options, expert testimony, grants or patents received or pending, or royalties.

No writing assistance was utilized in the production of this manuscript.

\section{References}

1. Japan Bioanalysis Forum. http://bioanalysisforum.jp/en

2. The Government of Japan. Realizing Society 5.0. www.japan.go.jp/abenomics/_userdata/abenomics/pdf/society_5.0.pdf

3. Wakamatsu A, Ochiai S, Suzuki E et al. Proposed selection strategy of surrogate matrix to quantify endogenous substances by Japan Bioanalysis Forum DG2015-15. Bioanalysis 10(17), 1349-1360 (2018).

4. Staack RF, Jordan G, Dahl U et al. Free analyte QC concept: a novel approach to prove correct quantification of free therapeutic protein drug/biomarker concentrations. Bioanalysis 6(4), 485-496 (2014).

5. US FDA Center for Drug Evaluation and Research. Bioanalytical method validation guidance for industry (2018). www.fda.gov/files/drugs/published/Bioanalytical-Method-Validation-Guidance-for-Industry.pdf

6. Kumagai Y, Aso M, Odagiri K et al. A checklist for early clinical trials [translation]. Jpn J. Clin. Pharmacol. Ther. 49(5), 183-194 (2018) (in Japanese). 